\title{
THE PARTICULARITIES OF ABPM PARAMETERS IN HYPERTENSIVE PATIENTS WITH NON-DIALYSIS CKD
}

\author{
Univ. Assist. Alexandru Minca ${ }^{1,2}$, MD, Mihai Comsa ${ }^{3}$, MD, Maria Mirabela Manea ${ }^{4}$, MD, \\ Univ. Assist. Maria Daniela Tanasescu ${ }^{1,2}$, MD, PhD, Lecturer Dorin Dragos ${ }^{1,2}$, MD, PhD, \\ Prof. Mircea Nicolae Penescu ${ }^{1,5}, \mathrm{MD}, \mathrm{PhD}$, Asoc. Prof. Dorin Ionescu ${ }^{1,2}, \mathrm{MD}, \mathrm{PhD}$ \\ 1 "Carol Davila" University of Medicine and Pharmacy, Bucharest \\ ${ }^{2}$ Nephrology Department, University Emergency Hospital, Bucharest \\ 3 "Nicolae C. Paulescu" National Institute for Diabetes, Nutrition and Metabolic Disease, Bucharest \\ ${ }^{4}$ Neurologic Clinic, National Institute of Neurology and Cerebrovascular Diseases, Bucharest \\ ${ }^{5}{ }^{\text {nd }}$ Nephrology Department, "Dr. Carol Davila” Clinical Hospital of Nephrology, Bucharest
}

\begin{abstract}
REZUMAT
Chronic kidney disease (CKD) affects approximately two million people (in a population of 20 million) in Romania. Hypertension is often associated with CKD and both (hypertension and CKD) are risk factors for cardiovascular (CV) events. Ambulatory blood pressure monitoring (ABPM) is increasingly used all around the world for the diagnosis and monitoring of BP (blood pressure) because it is proven that the ABPM is superior to office BP measurements in evaluating patients with hypertension, with or without CKD. Reduced nocturnal BP fall (non-dipping or reverse-dipping patterns) is associated with target organ damage, especially kidney disease and the proportion of non-dippers and reverse-dippers patients increases progressively with the reduction of glomerular filtration rate (GFR). Another ABPM parameter, ambulatory arterial stiffness index (AASI), is an index which was recently proposed for the evaluation of arterial stiffness (a better tool than PP). It has prognostic value for cardiac death and stroke and several studies have showed that is negatively related to eGFR and is positively related to albuminuria. Hyperbaric area index (HBI) might be considered a novel sensitive marker [independent of patterns of NBPC (nocturnal BP change)] for the reduction of kidney function. These facts suggest that ABPM offers multiple useful data with impact, not only in future CV and renal outcomes assessment, but also in the treatment and management of hypertensive patients with CKD.
\end{abstract}

Keywords: hypertension, ambulatory blood pressure monitoring, chronic kidney disease, cardiovascular risk, ambulatory arterial stiffness index, hyperbaric area index, pulse pressure

\section{INTRODUCTION}

It is known that kidney disease is the 9th leading cause of death in the United States (1). The prevalence of CKD in Romania is 9-12\%, similar to other European countries (2). Kidney Disease Improving Global Outcomes (KDIGO) has defined CKD as abnormalities of kidney structure or function, present for $>3$ months with major implications for health. The abnormalities include one or more markers of kidney damage (eg, albuminuria $>30$ $\mathrm{mg} / \mathrm{g}$ of creatinine, urine electrolyte or sediment abnormalities and other abnormalities due to tubular disorders, abnormalities detected by histology, history of kidney transplantation, or structural abnormalities detected by imaging) or a glomerular filtration rate (GFR) $<60 \mathrm{~mL} / \mathrm{min} / 1.73 \mathrm{~m}^{2}$ (GFR categories 3 a to 5) (3).

Approximately $80-85 \%$ of patients with CKD have hypertension and the proportion increases further as the GFR falls (4). Cardiovascular disease is the leading cause of death in patients with CKD (5).

The CKD patients are at increased risk of cardiovascular events. On the other hand, hypertension is often associated with $\mathrm{CKD}$, - it either causes $\mathrm{CKD}$, is a result of CKD, or is associated with CKD without evidence of a causal relationship. ABPM 
has become indispensable for the diagnosis and monitoring of hypertension in renal patients, because many studies have proven the superiority of ABPM to random office BP measurements, in patients with hypertension with and without CKD (6). Although ABPM is increasingly used all around the world for the diagnosis and monitoring of BP, in Romania it is only in its infancy.

This article aims to highlight the particularities of ABPM parameters in hypertensive patients with CKD.

Both hypertension and low GFR, are risk factors for $\mathrm{CV}$ events. Hypertension is considered the most important factor for CKD progression. For the diagnosis and monitoring of hypertension both office and home measurement may be used. ABPM requires the employment of a digital device and has become a powerful tool for the appraisal of hypertension since the late $80 \mathrm{~s}$. However, ABPM is not yet standardized, nor is its equivalence to office BP measurements established, as most validated studies on $\mathrm{CV}$ disease and renal disease have used office BP assessment.

\section{ABPM parameters and CKD}

Compared to office blood pressure (BP), the ABPM can offer more useful parameters for the management of CKD and for CV risk assessment: morning $\mathrm{BP}$; $\mathrm{BP}$ variability; ambulatory arterial stiffness index (AASI); nocturnal BP drop (diurnal index); pulse pressure, systolic/diastolic/mean BP, minimum/maximum; hyperbaric index (HBI).

Several recent international hypertension management guidelines (7-9) grant increasing trust to methods of measuring BP outside the medical environment, self-measurements at home (HBP), 24-hour ambulatory BP (ABP) measurements, or both. Indeed, mean BP over 24 hours is significantly better associated with morbidity and mortality (with respect to cerebrovascular or coronary events) than BP obtained in the office (10-12).

Normal values for ABPM are a daytime average of less than 135/85 $\mathrm{mmHg}$ and a night-time average of less than $120 / 70 \mathrm{mmHg}$, but even lower thresholds are advocated, particularly in high-risk groups, such as diabetic patients (13).

The existence of ,white-coat" normo-tension is well-known. The ABPM has identified patients in whom out-of-office BP is clearly much higher than office $\mathrm{BP}$, but the prevalence of this phenomenon is less well-known. Therefore, certain patients remain under-treated and are at higher risk for adverse CV outcomes. Elevated BP at night may be observed in some patients, also (14).
Multiple studies on CKD patients have demonstrated that $\mathrm{ABPM}$ and home $\mathrm{BP}$ measurement are better correlated with end-organ damage than office BP measurements. This is true for markers of kidney damage, particularily for proteinuria: a cross-sectional study of 232 CKD patients pointed out that $\mathrm{ABPM}$ is more strongly associated with proteinuria than office BP (15).

\section{Nighttime BP and CKD}

The only non-invasive technique which allows $\mathrm{BP}$ to be monitored during sleep is ABPM. There is increasing evidence that nighttime $\mathrm{BP}$ assessment can provide important information: diminished decrease of BP during night is associated with target organ damage, especially with CKD, and this may be a useful, albeit nonspecific, clue for secondary hypertension (renal hypertension). In addition, The Ohasama study demonstrated that the lack of nocturnal decline in $\mathrm{BP}$ is a risk factor for $\mathrm{CV}$ death (16).

Patients whose BP decreases at night are sometimes called "dippers" and those whose BP does not drop are also known as "non-dippers" (17).

The circadian BP profile is different in patients with CKD than in other patients, being marked by a preponderance of non-dippers and reverse-dippers (which is true not only for patients with renal hypertension, but also for those without hypertension). The lower the eGFR, higher this preponderance among renal patients. Several studies have shown that ABPM is more advantageous than office $\mathrm{BP}$ in evaluating the risk for both $\mathrm{CV}$ events and for CKD progression to ESRD and death. It seems that collecting data by ABPM can predict subsequent renal evolution and occurrence of $\mathrm{CV}$ events in patients with CKD (18).

There is an association between elevated nighttime BP, left ventricular (LV) hypertrophy, albuminuria and CKD (19) The riser BP pattern, or reverse-dipper, means that nocturnal $\mathrm{BP}$ exceeds daytime BP. A cross-sectional study that involved 10271 hypertensive patients found a large difference between the patients with and without CKD in the prevalence of the riser BP pattern $17.6 \%$ vs. $7.1 \%$, which means that CKD is associated with a 2.5 times higher prevalence of the BP pattern associated with the highest CVD risk. Moreover, among patients with CKD, the lower the GFR, the higher the prevalence of this high-risk BP pattern: $8.1 \%$ in stage $1 \mathrm{CKD}$ vs. $34.9 \%$ in stage 5 CKD (20).

Because of higher non-dipper BP profile in CKD patients, researchers tried to apply this find- 
ing in treatment management. Many trials have reported that adjusting the administration time for the blood pressure lowering medication significantly reduces $\mathrm{CV}$ events, bedtime administration being preferred to early morning (21). It is recomended to use ABPM for diagnosis and management of hypertension in all patients with CKD (22).

\section{Pulse pressure}

The pulse pressure (PP) is deffined as the difference between the systolic and the diastolic pressures. This value seems to be superior in predictive value to the systolic or diastolic values taken separately.

The higher PP increases the stress on arteries. This stress produces fatigue and an increased rupture rate in the elastic components of the arterial wall, increasing the risk of atherosclerosis and thrombosis (23).

In the Framingham Heart Study it was observed that an increment of $10 \mathrm{mmHg}$ in the PP was associated with a $23 \%$ increase in the risk of developing coronary heart disease (CHD) (24). This clear association of CHD with PP was visible in patients over the age of 50 years, and most evident in patients over the age of 60 years (25) and the adverse outcomes's association with higher PP observed in patients over the age of 50 years, appear to apply to hypertensive, as well as to normotensive patients, including those with normal systolic but low diastolic blood pressure (26).

Several researchers studied the relationship between PP and decreased GFR. It was observed that $\mathrm{PP}$ is an independent risk factor for progression of CKD (20,27-30,). In a post-hoc analysis of the RENAAL trial, 1513 patients with diabetic nephropathy were studied for the effects of BP level on the progression of diabetic nephropathy (29). After controlling for multiple potential confounders, a $17 \%$ higher relative risk of developing ESRD was associated with a $10 \mathrm{mmHg}$ increment in PP. A similar association was not found for diastolic pressure.In a study on 329 patients with CKD it was observed that a $10 \mathrm{mmHg}$ higher PP was significantly associated (after approximately 6 months of follow-up) with a $10 \%$ increase in relative risk of kidney function decline. This study observed that $\mathrm{PP}$ is a better predictor for progression of CKD (even for mild to moderate reductions in GFR) than DBP or even SBP (28).In a cross-sectional cohort of 2144 patients with CKD from the Chronic Renal Insufficiency Cohort (CRIC) study, Weir et al. (2011) found that PP, a correlate of the pulsatile he- modynamic load and conduit vessel stiffness, as well as an important CV risk factor, is also independently associated with urine protein excretion $(31,32)$. This association may explain why microalbuminuria predicts cardiovascular events in nondiabetic subjects. PP is not only a simple marker for atherosclerotic disease, but the independence from concomitant vascular disease of its association with proteinuria suggests that $\mathrm{PP}$ influences albuminuria directly (33).

\section{The ambulatory arterial stiffness index}

The AASI, derived from ABPM, is an index which was recently proposed for the evaluation of arterial stiffness (34).

On repeated measurements, the values of SBP and DBP follow a similar pattern, being linearly correlated to one another. However, for subjects with arterial stiffness, a given increase in DBP is associated with a greater increases in SBP, as a consequence of declining arterial compliance. This relationship can be assessed by scatter-plotting DBP against SBP (i.e. individual measurements obtained by means of ABPM) and calculating the linear regression slope of DBP on SBP, which is considered an overall measure of arterial capacitance while its complement (1 minus the regression slope) is used to assess arterial stiffness. The stiffer the arteries the closer is AASI to 1 (35). It is known that aging is an important determinant for arterial stiffness, therefore it is probable to exist a relationship between age and AASI.

The fact that AASI is an indirect arterial stiffeness marker is supported by one study published by Li et al. They found that AASI closely correlated not only with the peripheral and central systolic augmentation indices, but also with aortic pulse wave velocity (36).

In a cohort of 11,291 patients, Dolan et al. found that AASI has prognostic value for cardiac death and stroke (37).

One study on european subjects found that elevated AASI is a stronger predictor of stroke, beyond traditional CV risk factors, including PP and the mean arterial pressure (38). Presently, a great number of specialists propose that the software for ABPM analysis should include in the near future AASI as a marker in cardiovascular risk assessment.

Several studies have showed a correlation between AASI and renal function among hypertensive patients: hypertensive patients with CKD have a higher AASI than hypertensive patients without 
CKD $(39,40)$. Ratto et al. demonstrated that AASI is negatively related to eGFR and positively related to albuminuria (41).

CKD patients have stiffer arterial tree when compared to normal-creatinine-patients of similar age and BP $(42,43)$. Beyond hypertension and dyslipidemia, uremia itself plays a role in the stiffening process, maybe by mineral metabolism alterations and arterial calcification (44).

\section{Hyperbaric area index}

Hyperbaric area is defined as the area encircled by the line of ABPM and two boundary lines of hypertension limits: $135 / 85 \mathrm{mmHg}$ (during the day) and 120/70 $\mathrm{mmHg}$ (during the night). The HBI is described as 24-h adjusted hyperbaric area, an index of BP load (obtained by ABPM) on different organs. Since diastolic HBI is more affected by arteriosclerosis, it is not used frequently.

The CKD-JAC study, with a cohort of 2,977 japanese patients, founds that HBI might be considered a novel sensitive marker [independent of patterns of NBPC (nocturnal BP blood pressure change)] for the reduction of kidney function (45).

\section{Blood pressure variability}

Daily BP variability (BPV), obtained by a ABPM device, is considered more and more as an important risk factor for $\mathrm{CV}$ events occurence (with prognostic value for $\mathrm{CV}$ mobidity and mortality) and end-organ damage. It was shown that an enhanced variability in nocturnal SBP in initially untreated hypertensive subjects is an independent predictor of cardiac events.

In the past the $\mathrm{CV}$ consequences of hypertension were considered to be primarily determined by the average BP values. Nowadays however, multi- ple trials and observational studies have highlighted a possible important role of enhanced BPV in this regard. Measures of BPV can be obtained through different methods. In the Jackson heart study on African Americans, CKD was associated with higher 24-h BPV (using 24-h ABPM); however, after adjustment for mean 24-h BP, this association was no longer present. Several studies focusing on untreated essential hypertensive patients have shown that an increased short-term BPV is inversely correlated with GFR and is directly correlated with urinary albumin excretion.

\section{CONCLUSIONS}

The ABPM can help evaluate the risk of CKD progression to ESRD and death when added to office BP monitoring. The ABPM is mandatory for the adequate diagnosis and CVD risk assessment in hypertensive patients with CKD and may bring a contribution to establish the proper therapeutic scheme in order to diminish the long term CVD risk, although presently most treatment decisions use clinic BP values obtained by standard measurement methods.

In conclusion, it is strongly recomended to use ABPM for diagnosis and management of hypertension in all patients with CKD.

\section{Acknowledgements}

I would like to express the deepest appreciation to Professor Penescu Mircea-Nicolae, whose excellent personality continually demonstrates a high scientific spirit. His constant help makes this review possible. Also, my appreciations and thanks go to my colleagues in developing the project.

\section{REFERENCES}

1. Albright A., Burrows N.R., Jordan R., Williams D.E. The kidney disease initiative and the division of diabetes translation at the centers for disease control and prevention. Am J Kidney Dis. 2009; 53:S121S125. doi: 10.1053/j.ajkd.2008.06.037;

2. Covic A., Schiller A., Constantinescu O., Bredetean V., Mihaescu A., Olariu N. et al. Stage 3-5 chronic kidney disease what is the real prevalence in Romania? Revista Medico-Chirurgicala a Societatii de Medici si Naturalisti din lasi. 2008; 112(4):922-31;

3. Levey A.S, Eckardt K.W., Tsukamoto Y. et al. Definition and classification of chronic kidney disease: A position statement from Kidney Disease: Improving Global Outcomes (KDIGO) Kidney International Volume 67, Issue 6, June 2005, Pages 2089-2100;

4. Whaley-Connell A.T., Sowers J.R., Stevens L.A., McFarlane S.I., Shlipak M.G., Norris K.C., Chen S.C., Qiu Y., Wang C., Li S., Vassalotti J.A., Collins A.J. Kidney Early Evaluation Program
Investigators - CKD in the United States: Kidney Early Evaluation Program (KEEP) and National Health and Nutrition Examination Survey (NHANES) 1999-2004. SO Am J Kidney Dis. 2008; 51(4 Suppl 2):S13.);

5. Wright J., Hutchison A. Cardiovascular disease in patients with chronic kidney disease. Vasc Health Risk Manag. 2009; 5: 713-722. Published online 2009 Sep 7. PMCID: PMC2742701

6. Eguchi K., Pickering T.G., Hoshide S., Ishikawa J., Ishikawa S., Schwartz J.E., Shimada K., Kario K. Ambulatory blood pressure is a better marker than clinic blood pressure in predicting cardiovascular events in patients with/without type 2 diabetes. Am J Hypertens 2008; 21: 443-450;

7. Mancia G., De Backer G., Dominiczak A. et al. 2007 Guidelines for the Management of Arterial Hypertension: The Task Force for the Management of Arterial Hypertension of the European Society of 
Hypertension (ESH) and of the European Society of Cardiology (ESC). J Hypertens. 2007;25(6):1105-87. 10.1097/ HJH.0b013e3281fc975a;

8. Chobanian A.V., Bakris G.L., Black H.R., Cushman W.C., et al. Seventh report of the Joint National Committee on Prevention, Detection, Evaluation, and Treatment of High Blood Pressure. Hypertension. 2003; 42:1206-1252;

9. Ogihara T., Kikuchi K., Matsuoka H. et al. The Japanese Society of Hypertension guidelines for the management of hypertension (JSH 2009) Hypertension Research. 2009; 32(1):3-107;

10. Grossman E. Ambulatory Blood Pressure Monitoring in the Diagnosis and Management of Hypertension. Diabetes Care. 2013; 36 (Suppl 2): S307-S311. doi: 10.2337/dcS13-2039.

11. Gabbai F.B., Rahman M., Hu B. et al. Relationship between Ambulatory BP and Clinical Outcomes in Patients with Hypertensive CKD. Clinical Journal of the American Society of Nephrology: CJASN. 2012; 7(11):1770-1776. doi:10.2215/CJN.11301111;

12. Agarwal R., Andersen M.J. Prognostic importance of ambulatory blood pressure recordings in patients with chronic kidney disease. Kidney Int. 2006 Apr; 69(7): 1175-80;

13. O'Brien, E., Asmar, R., Beilin, L., Imai, Y., Mallion, J. M., Mancia, G., Parati, G. European Society of Hypertension recommendations for conventional, ambulatory and home blood pressure measurement. Journal of hypertension, 2003, 21(5), 821-848.

14. Schwartz G.L. Update on Noninvasive Ambulatory Blood Pressure Monitoring, FAC, 2nd Virtual Congress of Cardiology, set 1 - nov 30, 2001;

15. Agarwal R., Andersen M.J. Correlates of systolic hypertension in patients with chronic kidney disease. Hypertension. 2005; 46:514-520;

16. Ohkubo T., Hozawa A., Yamaguchi J., Kikuya M., Ohmori K., Michimata M. et al. Prognostic significance of the nocturnal decline in blood pressure in individuals with and without high 24-h blood pressure: the Ohasama study. J Hypertens (2002) 20(11):21839.10.1097/00004872-200211000-00017;

17. O’Brien E., Sheridan J., O'Malley K. Dippers and non-dippers. Lancet. 1988;2(8607):397;

18. Debbie L. Cohen, Yonghong Huan, Raymond R.T. Ambulatory blood pressure in chronic kidney disease. Curr Hypertens Rep. 2013 Jun; 15(3): 160-166. doi: 10.1007/s11906-013-0339-2;

19. Wang C., Deng W.J., Gong W.Y., Zhang J., Zhang Q.Z., Ye Z.C., Lou T. Nocturnal hypertension correlates better with target organ damage in patients with chronic kidney disease than a nondipping pattern. J Clin Hypertens (Greenwich). 2015; 17:792-801;

20. Mojón A., Ayala D.E., Piñeiro L., Otero A., Crespo J.J., Moyá A., Bóveda J., Pérez de Lis J., Fernández J.R., Hermida R.C. et al. Comparison of ambulatory blood pressure parameters of hypertensive patients with and without chronic kidney disease. Chronobiol Int. 2013 Mar; 30(1-2): 145-158. Published online 2012 Oct 25. doi: 10.3109/07420528.2012.703083;

21. Crespo J.J., Piñeiro L., Otero A., Castiñeira C., Rios M.T., Regueiro A., Mojón A., Lorenzo S., Ayala D.E., Hermida R.C. et al. Administration-Time-Dependent Effects of Hypertension Treatment on Ambulatory Blood Pressure in Patients With Chronic Kidney Disease. Chronobiol Int. 2013 Mar; 30(1-2): 159-175. Published online 2012 Oct 25. doi: 10.3109/07420528.2012.701459;

22. Agarwal R., Peixoto A.J., Santos S.F., Zoccali C. Out-of-office blood pressure monitoring in chronic kidney disease. Blood Press Monit. 2009 Feb; 14 (1): 2-11. doi: 10.1097/MBP.0b013e3283262f58;

23. Townsend R.R., Bakris G.L., Sullivan D.J., Forman J.P. Increased pulse pressure. Uptodate. Literature review current through: Jun 2017. This topic last updated: Jan 04, 2016;

24. Franklin S.S., Khan S.A., Wong N.D. et al. Is pulse pressure useful in predicting risk for coronary heart Disease? The Framingham heart study. Circulation 1999; 100:354;

25. Franklin S.S., Larson M.G., Khan S.A. et al. Does the relation of blood pressure to coronary heart disease risk change with aging? The Framingham Heart Study. Circulation 2001; 103:1245;

26. Thomas F., Blacher J., Benetos A. et al. Cardiovascular risk as defined in the 2003 European blood pressure classification: the assessment of an additional predictive value of pulse pressure on mortality. J Hypertens 2008; 26:1072;

27. Muntner P., Anderson A., Charleston J. et al. Hypertension awareness, treatment, and control in adults with CKD: results from the Chronic Renal Insufficiency Cohort (CRIC) Study. Am J Kidney Dis 2010; 55:441;

28. Arulkumaran N., Diwakar R., Tahir Z. et al. Pulse pressure and progression of chronic kidney disease. J Nephrol 2010; 23:189;

29. Bakris G.L., Weir M.R., Shanifar S. et al. Effects of blood pressure level on progression of diabetic nephropathy: results from the RENAAL study. Arch Intern Med 2003; 163:1555;

30. Tanaka M., Babazono T., Takeda M., Iwamoto Y. Pulse pressure and chronic kidney disease in patients with type 2 diabetes. Hypertens Res 2006; 29:345;

31. Weir M.R., Townsend R.R., Fink J.C. et al. Hemodynamic correlates of proteinuria in chronic kidney disease. Clin J Am Soc Nephrol 2011; 6:2403;

32. Kim J.K., Ju Y.S., Moon S.J. et al. High pulse pressure and metabolic syndrome are associated with proteinuria in young adult women. BMC Nephrol 2013; 14:45;

33. Pedrinelli R., Dell'Omo G., Penno G., Bandinelli S., Bertini A., Di Bello V., Mariani M. Microalbuminuria and pulse pressure in hypertensive and atherosclerotic men. Hypertension. 2000 Jan; 35(1 Pt 1):48-54;

34. Muxfeldt E.S., Fiszman R., Castelpoggi C.H., Salles G.F. Ambulatory Arterial Stiffness Index or Pulse Pressure: Which Correlates Better with Arterial Stiffness in Resistant Hypertension? Hypertension Research (2008) 31, 607-613; doi:10.1291/ hypres.31.607;

35. Acampa M., Guideri F., Di Donato I. et al. Arterial Stiffness in Patients with Deep and Lobar Intracerebral Hemorrhage. Journal of Stroke. 2014;16(3):184-188. doi:10.5853/jos.2014.16.3.184;

36. Li Y., Wang J.G., Dolan E., Gao P.J., Guo H.F., Nawrot T., Stanton A.V., Zhu D.L., O'Brien E., Staessen J.A. Ambulatory arterial stiffness index derived from 24-hour ambulatory blood pressure monitoring. Hypertension. 2006 Mar;47(3):359-64. Epub 2006 Jan 23;

37. Dolan E., L. Thijs, Y. Li et al. Ambulatory arterial stiffness index as a predictor of cardiovascular mortality in the Dublin outcome study," Hypertension, vol. 47, no. 3, pp. 365-370, 2006;

38. Hansen T. W., Staessen J.A., Torp-Pedersen C.B., Rasmussen S., Li Y., Dolan E., Thijs L., Wang Ji-G., O'Brien E., Ibsen H., Jeppesen J. Ambulatory arterial stiffness index predicts stroke in a general population. Journal of Hypertension: November 2006 Volume 24 - Issue 11 - p 2247-2253 doi: 10.1097/01. hjh.0000249703.57478.78;

39. Gismondi R.A., Neves M.F., Oigman W., Bregman R. Ambulatory arterial stiffness index is higher in hypertensive patients with chronic kidney disease. International journal of hypertension, 2012;

40. Mule G., Cottone S., Cusimano P. et al. Inverse relationship between ambulatory arterial stiffness index and glomerular filtration rate in arterial hypertension. American Journal of Hypertension, vol. 21 , no. 1 , pp. 35-40, 2008;

41. Ratto E., Leoncini G., Viazzi F. et al. Ambulatory arterial stiffness index and renal abnormalities in primary hypertension. Journal of Hypertension, vol. 24, no. 10, pp. 2033-2038, 2006;

42. Guerin A.P., Pannier B., Marchaisand S.J., London G.M. Arterial structure and function in end-stage renal disease. Current Hypertension Reports, vol. 10, no. 2, pp. 107-111, 2008;

43. Verbeke F.H., Agharazii M., Boutouyrie P., Pannier B., Guerin A.P., London G.M. Local shear stress and brachial artery functions in end-stage renal disease. Journal of the American Society of Nephrology, vol. 18, no. 2, pp. 621-628, 2007;

44. Guerin A.P., Pannier B., Metivier F., Marchaisand S.J., London G.M. Assessment and significance of arterial stiffness in patients with chronic kidney disease. Current Opinion in Nephrology and Hypertension, vol. 17, no. 6, pp. 635-641, 2008;

45. limuro S., Imai E., Watanabe T. et al. Hyperbaric area index calculated from ABPM elucidates the condition of CKD patients: the CKD-JAC study. Clinical and Experimental Nephrology. 2015;19(1):114-124. doi:10.1007/s10157-014-0965-2. 